\title{
A Storage and a Research Model of Medical Imaging in a Hospital Information System
}

\author{
Mohamad Ali Cheaito ${ }^{1}$, Marwan Nasri Cheaito ${ }^{2}$ \\ ${ }^{1}$ Lebanese University, Faculty of Science I, Computer Science Department, Hariri University Campus, Beirut-Hadath Lebanon, \\ ${ }^{2}$ Lebanese University, Faculty of science V, Computer Science Department, Nabatieh Lebanon
}

\begin{abstract}
The objective of our study is to focus on the domain of handling digital image [1] by a medical information system. The real evolution of the image is not recent but dates to the year 1963 with the project sketchpad of Ivan Sutherland [2] which shows the feasibility of creating, storing and manipulating computer images [3]. The construction process of digital image(IRM, CT, ...)[4], [5] pass by specific materials allowing toconvert the medical image informationinto a set of digital information stored in the computer machine[6].Thus, we need to transfer the medical image information into a computer which retainsthis information as a set of files by respecting the protocol of image conversion and the medium which manages the computer tool i.e. operating system.Afterwards, we must realize a system which permits the communication of medical image with a medical information system.The Database Management Systems (DBMS) are designed to manage textual information but makesdifficult the management of medical image. In our case, we are interested in the information system "CheBHosp"[7], [8] which uses a browser that allows the user to manipulate the medical image.
\end{abstract}

Keywords: Medical Image, Medical Information System, Patient, DBMS, DICOM, PACS

\section{Introduction}

Medical images are not limited to the process of scanning or digitalization, and they remain a complex task that is currently the subject of numerous studies. The nature of medical images requires taking new considerations in terms of capacity structuring, organization and storage. The management of medical images has been firstly highlighted by[9]. While medical images indexing is a difficult step to achieve on operating systemlevel, since most operating systems store medical images as a set of files defined by certain number of restricted information(name, extension, creation date, modification date,...). On the other hand, database management systems are indexing systems[10] whichmanage textual and numerical information but are not designed for the management of medical images.For this reason, we are interested in realizing a model that combines between the database management system and the operating system in order to facilitate the management and indexing of medical images.

\section{Medical image and information system}

The creation of the medical image[11] takes place in a hospital center by a technicianwhich sends the result of the medical image to a radiologist who manipulates and analyzes the image in order to write a descriptive report with conclusions which are necessary for the concerned doctor who must make a decision about them in order to be carried out by the nurse(s) of the department(figure 1)

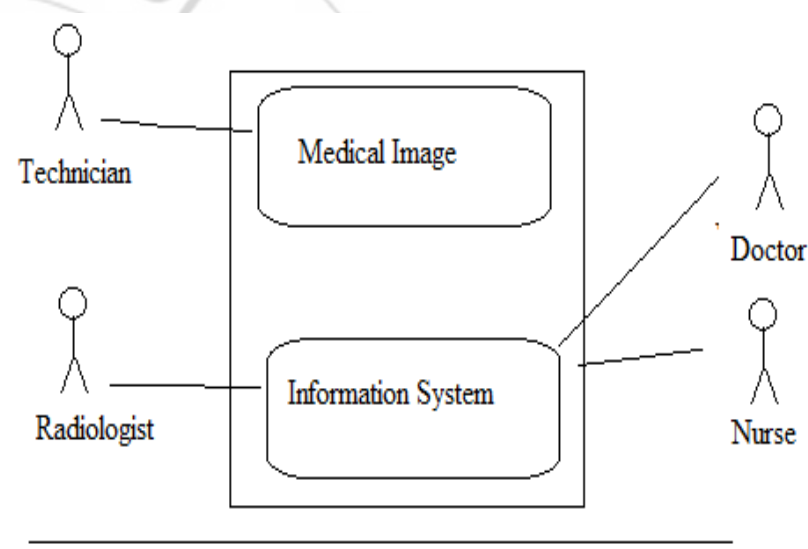

Figure 1: Use case diagram

The integration of medical images in a hospital information system has several advantages:

- Reducing the cost of image production. To avoid generating some non-usefulimages, there is a need for a tool for sharing medical images between physicians of different specialties and services.

- Quick access to images. The implementation of a solution for medical image management improves the analysis time and the study of images and therefore optimizes the diagnosis and the therapeutic strategy.

\section{DBMS and Medical Image}

The traditional Database Management Systems (DBMS)[12]are designed to manage textual or numerical information which suffer from some problems that make difficult the integration of images. Quoting:

The storage of images requires on one hand, a larger disk space and, on the other hand, a more advanced storage techniques [13] that are used for textual data. 


\section{International Journal of Science and Research (IJSR) \\ ISSN (Online): 2319-7064 \\ Index Copernicus Value (2013): 6.14 | Impact Factor (2015): 6.391}

Image searching remains an open reflection domain for the scientific community [14]. The general problem of image searching [15] circulates around four main axes:

1. What information we can extract from the image and how?

2. How to interpret the image?

3. What the user searches for?

4. How to formulate and interpret the user's needs?

\section{Link between information system and medical imaging}

A patient in an information system is generally identified by two identifiers:
One that describes the patient personal information, and the other describes the patient entry within the hospital whether an external patient (just to make some examination without having to stay in the hospital) or an internal patient (staying within the hospital)(figure 2)

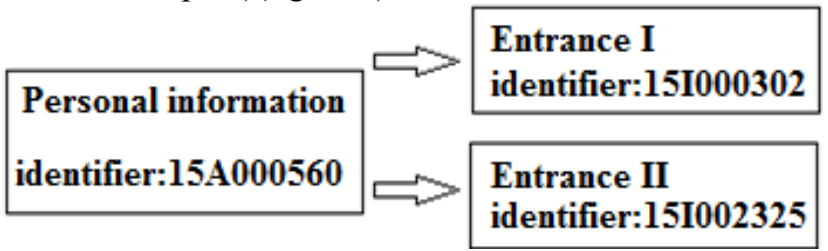

Figure 2: Patient identifiers

The patient entry identifier links the directory name which contains all the files representing the medical image of the patient (figure 3)

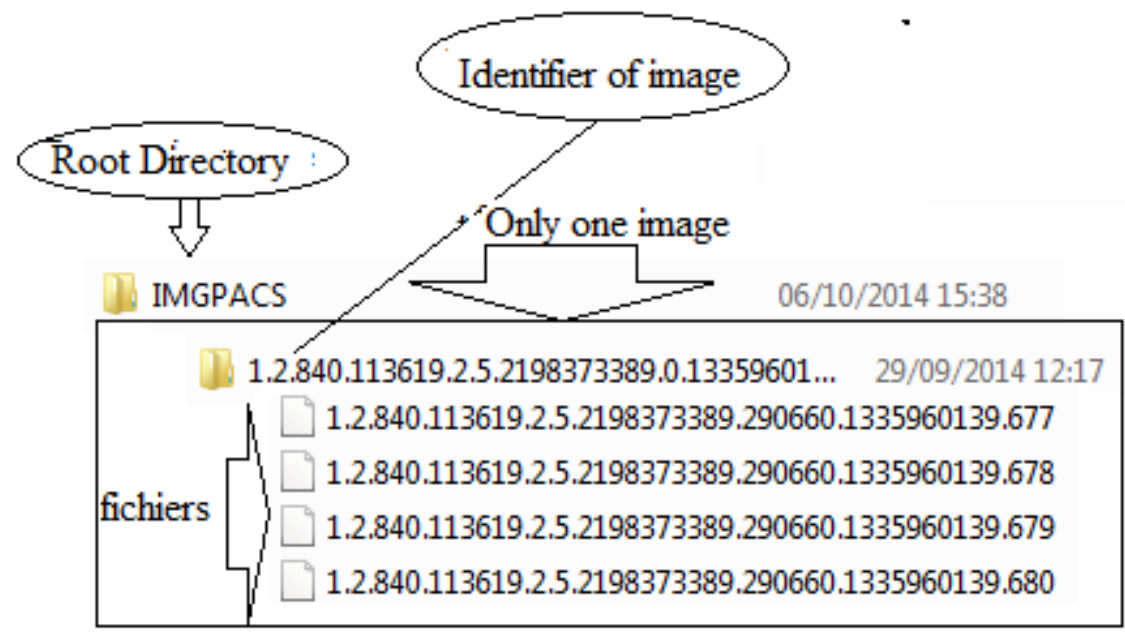

Figure 3: Medical image in an operating system using DICOM format

The link between all medical images and the hospital information system is described by the following entity nt:

RXRAY(ADMI,CARACT, SERIAL, CODE_TECH, COE_RADIOLOGUE, FDICOM, REPORT)

ADMI: is the identifier of the patient's case

CARACT: indicates image type (ECHO, SCANNER, IRM,..)

SERIAL :is an integer number so that ADMI, CARACT, SERIAL is an identifier of the entity RXARY and this identifier makes the link with other financial or medical image information.

CODE_TECH:is a codethat links technician information that are described in the HR (Human Resources) system.
CODE RADIOLOGUE:is a code that links radiologist information that are described in the HR (Human Resources) system.

REPORT: contains information of the written report by the radiologist.

FDICOM: contains information of the files identifiers of the medical image. So it makes the connection between the operating system and the hospital information system of the image information.

To realize the link between the operating system and the hospital information system the technician must enter the patient entry identifier of the file contents(ADMI) (figure 4)

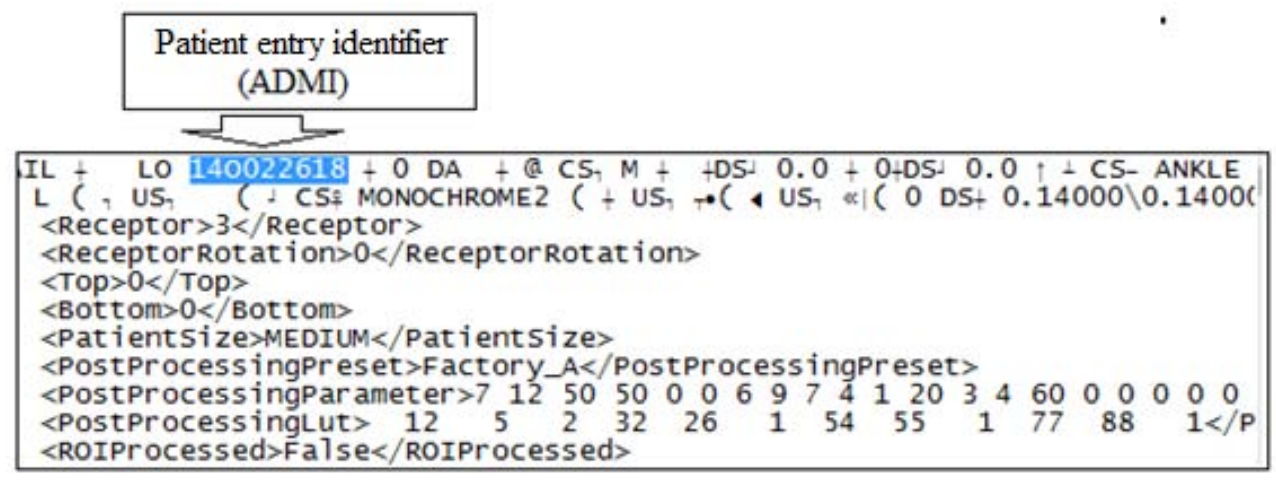

Figure 4: Notepad information describing medical image of identifier1400022618 


\section{International Journal of Science and Research (IJSR) \\ ISSN (Online): 2319-7064}

Index Copernicus Value (2013): 6.14 | Impact Factor (2015): 6.391

The medical image can be displayed by the software the technician (figure 5)

"UniViewer" and we can view the information entered by

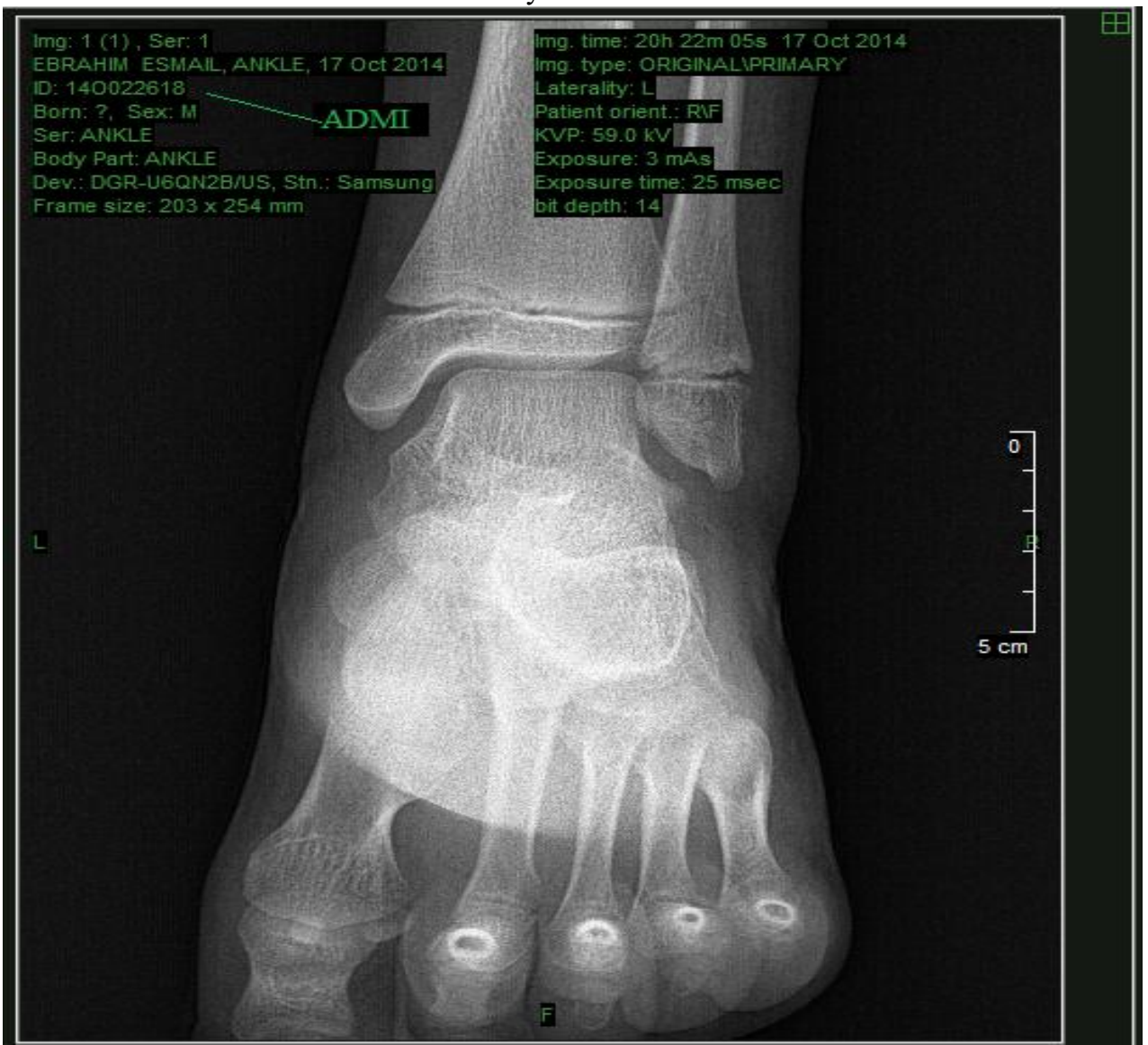

Figure 5: Entered information by technician (ADMI)

\section{The Search Interface}

The user must enter the necessary information in order the system to retrieve the patient medical image with the minimum time.For this, the user submits the reference and the date estimate limits (from date - to date) for the creation of the image (figure 6). The date estimate method allows to select all the files having their creation date between the two entered dates based on the following operating system command (ADIR(TP1, '*.*', 'D'))

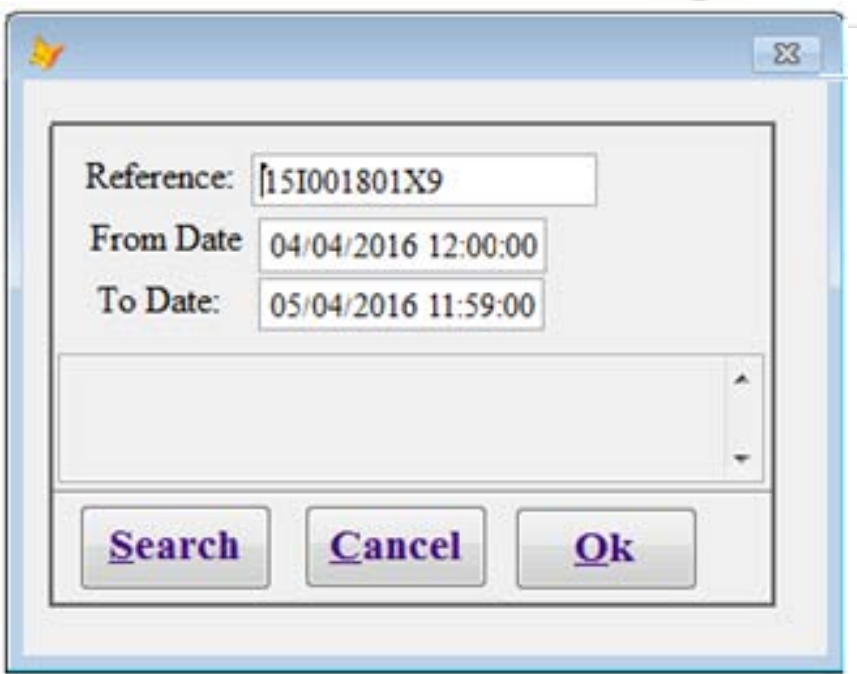

Figure 6: Required information to retrieve the patient's medical image

\section{The proposed algorithm to retrieve patient information}

FUNCTION CherchDemFile

Test $=$ False

MaAdmi=""

File=FOPEN("IMAGE.DCM",10)

DO WHILE((Not Test) AND (!EOF(File)))

$\mathrm{C} 1=\mathrm{FREAD}($ File, 1$)$

$\mathrm{IF}(\mathrm{C} 1=$ 'L' AND Not Test $)$

$\mathrm{C} 1=$ FREAD $($ File, 1$)$

$\mathrm{C} 2=\mathrm{FREAD}($ File, 1$)$

$\mathrm{C} 3=\mathrm{FREAD}($ File, 1$)$

$\operatorname{IF}\left(\mathrm{C} 1={ }^{\prime} \mathrm{O}^{\prime}\right.$ AND $(\mathrm{ASC}(\mathrm{C} 2)=10$ OR ASC $(\mathrm{C} 2)=4$ OR

$\mathrm{ASC}(\mathrm{C} 2)=2$ OR

$\operatorname{ASC}(\mathrm{C} 2)=6$ OR ASC (C2) $=12) \operatorname{AND~ASC}(\mathrm{C} 3)=0)$

Test $1=$ True

Test $=$ True

DO WHILE(Test1 AND (!EOF(File))

$\mathrm{C} 1=\mathrm{FREAD}($ File, 1$)$

$\operatorname{IF}(\mathrm{ASC}(\mathrm{C} 1) !=16)$

ELSE

$\mathrm{MaAdmi}=\mathrm{MaAdmi}+\mathrm{C} 1$

Test $1=$ False

ENDIF

ENDDO

ELSE

FSEEK(File,-3,1)

ENDIF

Volume 5 Issue 5, May 2016 


\section{International Journal of Science and Research (IJSR)}

ISSN (Online): 2319-7064

Index Copernicus Value (2013): 6.14 | Impact Factor (2015): 6.391

\section{ENDIF \\ ENDDO \\ $=$ FCLOSE(File) \\ ENDIF \\ RETURN MaAdmi}

\section{Conclusion}

With the advance of technology, the integration of medical images in the database management systems has become a necessity in several domains. In fact, thousands of images are daily produced by medical equipment's, so the usage of medical images requires new methods of storing, organizing, searching and browsing images. Since 70s, image search has experienced an important research and study activity. Most of the proposed solutions are based on highly correlated parameters of a chosen application domain and the expressed user's needs. In this domain, existing solutions, are often called PACS (Picture Archiving and Communication Systems), are only based on limited parameters such as patient name, and the date of image acquisition. In response to this problem, we have introduced a model that permits to store the image on the hard disk. Moreover, we have made a link through a special search that permits sharing the time by doing its link with the medical information system by conserving the directory name containing all the files of the medical image. These files play an important role in describing the concerned patient medical image and by a simple launch of the browser (UniViewer) we can access this image in order to write a text report of all the information detected by the radiologist.

\section{References}

[1] N. C.Dalrymphe,S. RPrasad, M. W. Freckleton, 2005: Introduction to the language of three-dimensional Imaging with Multidetecctor CT. Informatics in Radiology. Radiographics, 2005, 25(5), pp. 1409-1428.

[2] I.E., Sutherland, 1963: "SketchPad: A Man-Machine Graphical Communication System", Proceedings AFIPS Spring Joint Computer Conference, Detroit, MI., 1963, Vol. 23, P. 329-346

[3] R, Chbeir, Y. Amghar, A. Flory, 2000: "Image Modeling for Medical Databases", Proceedings of the ISCA 15th International Conference Computers and Their Applications CATA-2000, New Orleans, Louisiana USA, 2000, P. 24-28.

[4] J. Lepagnot, A. Nakib, H. Oulhadj, P. Siarry, 2013: Elastic registration of brain cine-MRI sequences using MLSDO dynamic optimization algorithm. In E Alba, A. NAakib\& P. Siarry (Eds), Metaheuristics for Dynamic Optimization, Springer, pp 211-224, 2013.

[5] J. Lepagnot, A. Nakib, H. Oulhadj, P. Siarry. 2011:Brain Cine MRI Segmentation Based on a Multiagent Algorithm for Dynamic Continuous Optimization. In Proceedings of the IEEE Congress on Evolutionary Computation, vol. 1, pp. 1695-1702, New Orleans, LA, USA, June 2011.

[6] D. A. Karras, S. A. Karkanis, D. E. Maroulis, 2000: "Efficient Image Compression of Medical Images Using the Wavelet Transform and Fuzzy c-means Clustering on Regions of Interest", Proceedings of The 26th
EUROMICRO Conference, 2000, Los Alamitos, CA, USA, Vol. 2, P. 469-473.

[7] M.A. Cheaito, M. N.Cheaito 2015: The Concept of a Medical Information System By Rate of Use (CheBHosp), Health Informatics-An International Journal (HIIJ), Vol 4, No 1/2,May 2015, Page:23-33, DOI:10.5121/hiij.2015.4201

[8] M. A. Cheaito , M. N.Cheaito 2015: Concept of Graphical Interface of a Medical Information System (CheBHosp) , International Journal of Science and Research (IJSR) ISSN (Online): 2319-7064 Index Copernicus Value (2013): 6.14 | Impact Factor (2014): 5.611, Volume 4, Issue 12, December 2015, Page:156159

[9] R. Jain, 1993: "NSF Workshop on Visual Information Management Systems", Proceedings of the SPIE - The International Society for Optical Engineering, 1993, Vol. 1908, P. 198-218.

[10]C. Berrut, 1988; "Une méthode d'indexation fondée sur l'analyse sémantique de documents spécialisés. Le prototype RIME et son application à un corpus medical", Thèse de doctorat de 3 cycle, Université de Grenoble, 1988, 206 p.

[11]T. Mickaël 2013 : unité 979 Inserm/CNRS - Université Paris-Diderot Paris 7 Ultrasons elastographie. JL Gennisson et al. DiagnInterv Imaging., mai 2013 ; 94 (5) : 487-95

[12] S. J. Cannan, 1992: "SQL - The Standard Handbook", London: McGraw Hill, 1992, 584 p.

[13]P. Suapang, K. Dejhan; S.Yimmun2010 : "Medical Image Archiving, Processing, Analysis and communication System for Teleraiology" TENCON 2010-2010 IEEE Region 10 Conference Year 2010 Pages:339-345, DOI:10.1109TENCON.210.5686025

[14]C. Daniel, E. Albuisson, P. Avillach, A. Burgun, G. Chêne, M. Cuggia,M. Goldberg, 2013 : Informatique biomédicale et recherche en santé Informatique translationnelle. In Informatique médicale, e-Santé (pp. 463-493). Springer Paris

[15] W. Yang; J.R.Liu2013: "Research and development of medical image fusion", Medical Imaging Physics and Engineering (ICMIPE), 2013 IEEE International Conference on Year 2013 Pages: 307-309, DOI:10.1109/ICMIPE.2013.6864557 